\title{
Information Processing During Transient Responses in the Crayfish Visual System
}

\author{
Christopher J. Rozell ${ }^{\mathrm{a}, 1}$, Don. H. Johnson ${ }^{\mathrm{a}}$ and Raymon M. Glantz ${ }^{\mathrm{b}}$ \\ ${ }^{\mathrm{a}}$ Department of Electrical \& Computer Engineering \\ ${ }^{\mathrm{b}}$ Department of Biochemistry \& Cell Biology
}

Rice University, Houston, TX 77251-1892

\begin{abstract}
We analyzed sustaining fiber responses in the crayfish visual system to light pulses using information processing techniques. The light pulse stimuli elicited a transient and a steadystate component in the EPSP input and in the firing rate of the spike train output. The overall information transfer of the system was very low $\left(\sim 10^{-4}\right)$, with a sharp increase during the transient portion of the response followed by a steady decrease. The information transfer dynamics are consistent with a simple spike generator model that depends explicitly on stimulus changes. The present analysis also corroborates the observed light reflex behavior.
\end{abstract}

Key words: Neural information processing; spike generator efficiency; crayfish visual system

\section{Introduction}

Sensory neurons generally encode some aspects of the information present in a stimulus and suppress others. Understanding and characterizing a sensorineural system's information processing capabilities in the presence of different stimuli is critical to progress in determining the coding structures underlying neural communication. Recently, a new theory of information processing (reviewed in section 2) has been presented that draws from classical information theory and statistical signal processing to provide a framework for quantifying the information processing characteristics of a general system [2]. We present an information processing analysis of crayfish sustaining fiber transient responses.

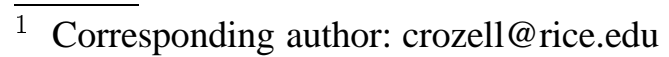

2 This work was supported by grant MH60861 from the National Institute of Mental Health and by grant CCR-0105558 from the National Science Foundation. 


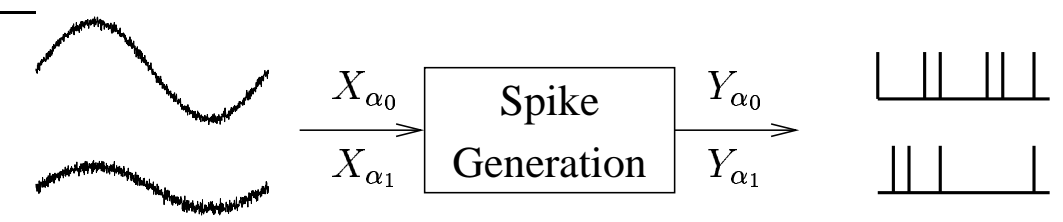

Fig. 1. The SF spike generator has an analog EPSP input $\left(X_{\alpha}\right)$ and a spike train output $\left(Y_{\alpha}\right)$ that both depend on the value of the input stimulus $(\alpha)$.

The retina of the crayfish compound eye has groups of photoreceptors projecting down the visual pathway in a 1:1 fashion to a column of non-spiking interneurons in the medulla. These retinotopically organized interneurons synapse on sustaining fiber (SF) dendrites [3]. SF input EPSPs closely resemble graded and delayed versions of summed photoreceptor activity over a segment of the visual field. The SFs are critical from an information processing perspective because they are the first stage where an analog signal representing an outside stimulus is converted to a discrete spike train. From a signal processing point of view, the SFs represent a "front-end" for later information processing in higher centers of the brain. The fundamental problem that we address here is quantifying the efficiency (and dynamic properties) of the spike generation process.

\section{Information Processing Analysis}

The analysis framework presented in [2] is based on the concept of presenting a system with two different stimulus conditions (denoted $\alpha_{0}$ and $\alpha_{1}$ ). The input EPSP signals and output spike trains are stochastic processes (denoted $X_{\alpha}$ and $Y_{\alpha}$ respectively, and depicted in figure 1) that have probability laws depending explicitly on the value of the stimulus. By examining how the input and output signals change when the stimulus values are changed, we can quantify how much information about that stimulus change is present in the signals. Because the signals are stochastic processes, we measure the distance between the probability laws that govern the input signals $\left(d_{X}\left(\alpha_{0}, \alpha_{1}\right)\right)$ and the spike train signals $\left(d_{Y}\left(\alpha_{0}, \alpha_{1}\right)\right)$ under both stimulus conditions. There are several information theoretic distances that measure changes in probability distributions. We use the Kullback-Leibler (KL) distance [1], which is relevant because it is the error decay rate for an optimal detector trying to distinguish the two stimuli conditions. The ratio of the output to input distances is termed the "information transfer ratio", $\gamma_{X, Y}\left(\alpha_{0}, \alpha_{1}\right)=\frac{d_{Y}\left(\alpha_{0}, \alpha_{1}\right)}{d_{X}\left(\alpha_{0}, \alpha_{1}\right)}$. The information transfer ratio is a single quantitative measure of the information processing efficiency of the system for the stimulus change under consideration. A fundamental result from information theory states that (for a large class of distances) the information transfer ratio will always be between zero and one $\left(0 \leq \gamma_{X, Y}\left(\alpha_{0}, \alpha_{1}\right) \leq 1\right)$. A value of one represents perfect information transfer of the stimulus change and a value of zero represents total information loss. 


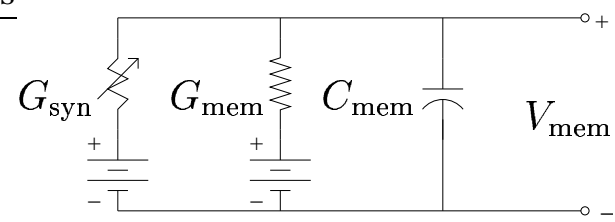

Fig. 2. The cell membrane acts as a time-varying, first-order lowpass filter.

In practice, the information transfer ratio is straightforward to estimate for the SFs. Two stimulus conditions $\left(\alpha_{0}, \alpha_{1}\right)$ are presented repeatedly and the EPSP input and spike train output responses are captured through intracellular recording. The repeated trials are used to estimate the probability law governing the responses under each stimulus condition. Simple histogram estimators are formed from the binned spike responses to both stimuli and KL distances are easily calculated using these estimates. Distance estimation using spike responses has been discussed previously in [2]. The EPSPs are inherently analog signals, and a non-parametric estimate of the probability law is impossible. The compound EPSP in the SF spike generation area is the sum of many discrete events, suggesting that a Gaussian model may be a good match (which is true in our SF data). If the sampled EPSP responses under each stimulus condition are given as Gaussian random vectors with different means and variances $\left(X_{\alpha_{0}} \sim N\left(m_{\alpha_{0}}, K_{\alpha_{0}}\right), X_{\alpha_{1}} \sim N\left(m_{\alpha_{1}}, K_{\alpha_{1}}\right)\right)$, the KL distance between them will be approximately given by

$$
d_{X}\left(\alpha_{0}, \alpha_{1}\right) \approx \frac{\left(m_{\alpha_{0}}-m_{\alpha_{1}}\right)^{t} K_{\alpha_{1}}^{-1}\left(m_{\alpha_{0}}-m_{\alpha_{1}}\right)}{2}
$$

The mean vectors $m_{\alpha_{0}}$ and $m_{\alpha_{1}}$ are easily estimated by simply averaging the repeated EPSP responses under each stimulus condition. However, equation (1) indicates that we also need the term $K_{\alpha_{1}}^{-1}$. In short, we need to know not only the power but also the correlation structure of the membrane noise.

At rest, the biophysical properties of the impermeable cell membrane together with the embedded ion channels act electrically as as capacitor $\left(C_{\mathrm{mem}}\right)$ in parallel with a resting membrane conductance $\left(G_{\mathrm{mem}}\right)$. It is also known that EPSP variations are a result of a variable synaptic conductance $\left(G_{\mathrm{syn}}\right)$, and the relationship between the amount of the depolarization in the SF and the increase in membrane conductance has been measured by Waldrop and Glantz [5]. During the generation of an EPSP the membrane acts as the time-varying first-order lowpass filter shown in figure 2. Using the mean EPSPs $\left(m_{\alpha_{0}}\right.$ and $m_{\alpha_{1}}$ ) and the results given in [5], the timevarying increase in total conductance for the SF can be calculated. Techniques from time-varying linear system theory given an analytic expression for the correlation structure of the membrane noise and the KL distance between EPSP responses under the two stimuli conditions follows. More details about the membrane model and the correlation calculations can be found in [4]. 


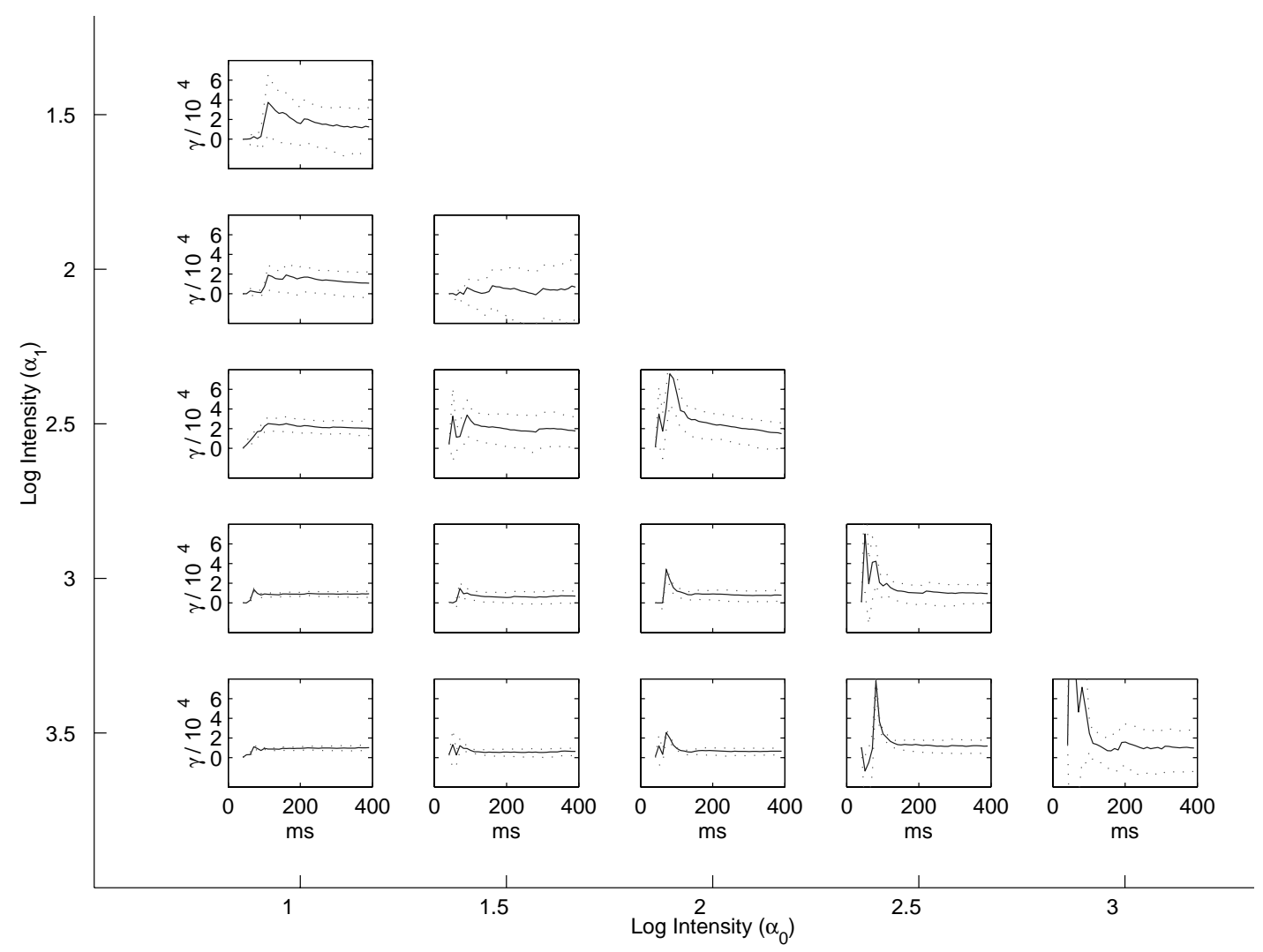

Fig. 3. Information transfer ratios for a SF. Outer axis are the log intensity stimuli values, and inner plots show time-varying information transfer ratios for a specific pair of stimuli.

\section{Results}

Several recordings were made from a crayfish sustaining fiber when the stimulus was a square wave pulse of light with intensity varying from trial to trial. At the stimulus onset, the input EPSP exhibits a transient pulse of high potential for approximately $100 \mathrm{~ms}$ followed by a decrease to a plateau which is maintained for the duration of the stimulus. The output spike trains also exhibit a transient burst of high firing rate, followed by an adaptive response that reduces the firing rate to a steady-state value maintained for the stimulus duration. The procedures outlined in section 2 were used to calculate KL distances between EPSP and spike responses for pairs of stimuli conditions. The time-varying information transfer ratios are shown for this dataset in figure 3, where the outer axis gives the log intensity of the stimuli values, and the inner plots show the time-varying information transfer ratio for a specific pair of stimuli. Note that the stimuli pair shown in the lower left corner represents the largest net stimulus change and the stimuli pairs along the diagonal (toward the upper right) have the smallest net stimulus change.

The first feature of figure 3 to notice is the scale of the plots. The information transfer ratio estimates have steady-state values on the order of $10^{-4}$. The low value of the information transfer indicates that a large portion of the information about 
the stimulus change that is present in the analog EPSP inputs is not present in the spike train outputs. Very low information transfer values on the order seen here are also observed in other SF units and when changing other stimulus features (such as spatial frequency). A single spike train can only communicate information with each action potential, and the low information transfer ratios indicate that the low spike rates seen in crayfish SFs (often $<20 \mathrm{~Hz}$ ) are inefficient compared to the high SNR analog EPSP inputs. The other feature noticeable in figure 3 is the general trend for information transfer ratios to often increase sharply after the stimulus onset followed by a slow decrease to a steady-state value. The sharp jump indicates that the system is transferring stimulus change information more efficiently when the spike rates burst to higher values, but the maximum communication rate is not sustainable when the rates fall back to steady-state levels.

We also used a simple spike generator model to investigate how information processing characteristics of a generic spike generator would depend on the properties of the encoding process. In our spike generator model, the input is a Gaussian random process $(X(t) \sim N(m(t), K))$ and the output is a Poisson random process $(Y(t) \sim \operatorname{Poiss}(\lambda(t)))$ where the rate depends on the input values $X(t)$. The output firing rate is given by an expression that depends explicitly on changes in the input:

$$
\lambda(t)=G_{s} X(t)+G_{t} \frac{d X(t)}{d t} .
$$

Using recorded SF inputs as $X(t)$ and estimating the steady-state and transient gains $\left(G_{s}, G_{t}\right)$ to roughly match the firing rates seen in the SF, we are able to analytically calculate the information transfer ratio of the model system (detail of the calculations is given in [4]). When using a firing rate that explicitly depends on changes in the input, the information transfer ratio for the model (not shown) has a similar transient behavior to that observed in the SF data. The transient behavior is not a dominant feature when the output rates depend only on the value of the input (and not explicitly on input changes).

\section{Conclusions}

An information processing analysis of a crayfish SF responding to sudden-onset light stimuli is presented. The information transfer ratios are on the order of $10^{-4}$ regardless of the actual stimulus change, and reflect the inefficiency of a low-rate spike train in representing the analog EPSP signal. The information transfer ratio appears to have dynamic properties, with the SF transferring information more efficiently during the transient the response. When also observing the elicited light reflex of the crayfish to the stimulus, all of the information from the stimulus producing the reflex behavior must be communicated through the sustaining fiber during the first $100 \mathrm{~ms}$ after the stimulus onset. The reflex behavior is therefore also consistent with the dynamics of the information processing analysis presented here. 
Finally, a simple spike generation model is presented where we can analytically calculate the information transfer ratio. When the output firing rates depend only on the value of the input, the information transfer ratio is not dominated by a transient and steady-state component. When the output firing rate also depends explicitly on the dynamic properties of the input, we observe the same time-varying behavior seen in the SF data. Therefore the information transfer dynamic properties of the SF are consistent with a system that is tuned for detecting changes in a stimulus rather than absolute values.

\section{References}

[1] T.M. Cover and J.A. Thomas. Elements of Information Theory. John Wiley \& Sons, Inc., New York, NY, 1991.

[2] D.H. Johnson, C.M. Gruner, K. Baggerly, and C. Seshagiri. Information-theoretic analysis of neural coding. J. Comp. Neuroscience, 10:47-69, 2001.

[3] M.D. Kirk, B. Waldrop, and R.M. Glantz. The crayfish sustaining fibers, I. Morphological representation of visual receptive fields in the second optic neuropil. Journal of Comparative Physiology, 146:175-179, 1982.

[4] C.J. Rozell. Analyzing dynamics and stimulus feature dependence in the information processing of crayfish sustaining fibers. Master's thesis, Rice University, Houston, TX, April 2002.

[5] B. Waldrop and R.M. Glantz. Synaptic mechanisms of a tonic EPSP in crustacean visual interneurons: Analysis and simulation. Journal of Neurophysiology, 54(3):636650, 1985.

Christopher J. Rozell received the M.S. in electrical engineering from Rice University in May 2002 and is currently in the Ph.D. program at Rice, with research interests in statistical signal processing applied to sensorineural systems.

Don H. Johnson received the S.B. and S.M. degrees in 1970, the E.E. degree in 1971 and Ph.D. in 1974, all in electrical engineering from the Massachusetts Institute of Technology (MIT). He is currently Professor and Chair of the Department of Electrical and Computer Engineering at Rice University. His current research areas are statistical signal processing and representation of information by neural signals.

Raymon M. Glantz received his doctorate from Syracuse University in 1967. After postdoctoral work at Caltech, he joined the faculty at Rice University in 1969, where he is currently Professor in the Department of Biochemistry and Cell Biology. His current research interests focus on information processing in the crayfish visual system. 